\title{
Operative Hektik
}

In der letzten regulären Sitzung dieser Legislaturperiode hat der Deutsche Bundestag am 3. Juli 2009 den „Entwurf eines Gesetzes zur Regelung des Datenschutzaudits und zur Änderung datenschutzrechtlicher Vorschriften" der Bundesregierung vom 18. Februar 2009 (Bundestagsdrucksache 16/12011) mit den Änderungsempfehlungen des Innenausschusses (Bundestagsdrucksache 16/13657) angenommen. Damit wurde im Hau-Ruck-Verfahren in letzter Minute auf die teilweise vernichtende Kritik selbst engagierter Verfechter eines gesetzlich geregelten Datenschutz-Audits reagiert. Der Innenausschuss, an den der Gesetzentwurf am 19. März 2009 vom Deutschen Bundestag federführend überwiesen worden war, hatte - offenbar unter dem Eindruck der erheblichen Einwände von Fachexperten in der öffentlichen Anhörung vom 23. März 2009 sowie von Seiten des Bundesrats - nach abschließender Beratung am 01. Juli 2009 empfohlen, eine Beschlussfassung über das Datenschutzauditgesetz zu Gunsten eines dreijährigen Pilotprojekts für eine Branche auszusetzen. Zwar muss man sich fragen, welche Art von Erkenntnis wohl von einem solchen beschränkten Pilotprojekt zu erwarten sein kann, die über die achtjährige Erfahrung des ULD Schleswig-Holstein mit Audit-Projekten hinausgeht. Immerhin war es eine elegante und wohlfeile Methode, dem festgefahrenen Karren unter einer neuen Bundesregierung eine neue Chance zu geben.

Auch wenn beklagt wird, dass es damit wieder nicht gelungen ist, ein Audit-Gesetz auf den Weg zu bringen - das Resultat wäre anderenfalls ein Gesetz gewesen, das niemand wirklich haben wollte, mit heißer Nadel gestrickt, voller handwerklicher Mängel im Detail und ohne realistische Perspektive, den Datenschutz in Deutschland wirksam zu verbessern. Eins wäre den Reaktionen auf ein solches Gesetz gemein gewesen: das klagende "ceterum censeo", dass mit etwas mehr Bedacht und kompetenter Analyse eine erheblich bessere Regelung möglich gewesen wäre. Zumindest am Ende einer Legislaturperiode ist das Risiko groß, dass die tatkräftige Reaktion auf Vorfälle und Skandale zum politischen Handlungsmaßstab wird - und mit einem Seitenblick auf die Wähler operative Hektik kundige Konzeption ersetzt.

Der nun beschlossene Rumpf-Gesetzentwurf betrifft verschiedene Regelungen des BDSG. So ist der betriebliche Datenschutzbeauftragte zukünftig nur noch „aus wichtigem Grund ohne Einhaltung einer Kündigungsfrist" kündbar, und die verantwortliche Stelle wird verpflichtet, die Teilnahme an Fort- und Weiterbildungsmaßnahmen zu ermöglichen und zu finanzieren. Die Zulässigkeit der Nutzung listenmäBig zusammengefasster Daten für Zwecke des Adresshandels bleibt erhalten, allerdings ist die für die Nutzung der Daten verantwortliche Stelle deutlich zu kennzeichnen und Herkunft sowie Empfänger der Daten sind über zwei Jahre für etwaige Auskunftsersuchen Betroffener zu speichern. Eine wesentliche Präzisierung hat die Auftragsdatenverarbeitung in § 11 BDSG erfahren - ein wichtiger Schritt, der zweifellos in zahlreichen Fällen eine Umformulierung bestehender Datenverarbeitungsverträge erforderlich machen wird. Konsequenter geregelt ist die Überprüfung der Verarbeitung durch den Auftragnehmer: Sie hat vor Beginn der Verarbeitung und regelmäßig während dessen Verlaufs zu erfolgen; die Ergebnisse sind zu dokumentieren. Als neuer $\S 32$ wurde eine Regelung zum Arbeitnehmerdatenschutz ergänzt - gut gemeint, aber inhaltlich nicht mehr als das, was ohnehin bereits geltendes Recht ist.

Die im Entwurf der Bundesregierung enthaltene Erhöhung der Geldbußen-Grenzen auf bis zu $50.000 €$ (bisher $25.000 €$ ) bzw. $300.000 €$ (bisher $250.000 €$ ) wurde hingegen gestrichen. Immerhin hätte eine solche Erhöhung eines unmissverständlich klar gestellt: Die Zeit, in der Datenschutzverstöße als Kavaliersdelikt durchgehen, ist endgültig vorbei.

\section{Dirk Fox}

